\title{
The Ideal Surgical Anaesthesia: A Comparison of Pain Experience in Subjects Undergoing Cataract Surgery Under Topical Versus Subtenon Anaesthesia
}

\author{
Teh SS, Abdul Ghani NA, Subrayan V, Ratanam M* and Peyman MR
}

Department of Ophthalmology, Hospital Sultan Haji Ahamd Shah (HoSHAS), Temerloh, Pahang, Malaysia

${ }^{*}$ Corresponding author: Mohanarasan Ratanam, Department of Ophthalmology, Hospital Sultan Haji Ahamd Shah (HoSHAS), Temerloh, Pahang, Malaysia Tel: +60 9-295 5333; E-mail: mohanslayer@gmail.com

Received: June 22, 2017; Accepted: July 05, 2017; Published: July 15, 2017

Citation: Teh SS, Abdul Ghani NA, Subrayan V, Ratanam M, Peyman MR (2017) The Ideal Surgical Anaesthesia: A Comparison of Pain Experience in Subjects Undergoing Cataract Surgery Under Topical Versus Subtenon Anaesthesia. Ann Clin Lab Res. Vol.5:No.3:181

\section{Abstract}

Background: Recent advancements in cataract surgery and local anaesthesia administration has led to the majority of cataract surgeries been performed under local anaesthesia. This has further intensified the search for the ideal anaesthesia. The use of topical anaesthetic agents in cataract surgeries has brought us closer to finding that ideal anaesthesia.

Objective: The objective of this study is to determine the viability of topical anaesthesia as a comparable mode of anaesthesia to subtenon anaesthesia in cataract surgery.

Methods: This is a randomized double-blinded placebocontrolled clinical trial. 118 subjects undergoing small incision cataract surgery were allocated to either a topical anaesthesia group (topical anaesthesia with placebo subtenon's injection of balanced salt solution, $n=40$ ) or subtenon's group (sub-tenon's anaesthesia with placebo topical balanced salt solution, $n=78$ ). Pain experienced during and after the operation was assessed using the numerical rating scale, aided with a visual analogue graphic pain score chart.

Results: There was no significant difference in mean pain score between the two groups during anaesthesia administration $(P=0.48)$, during cataract surgery $(P=0.37)$, immediately after surgery $(P=0.77)$ and 30 minutes after surgery $(P=0.17)$.

Conclusion: Topical anaesthesia is comparable to subtenon's anaesthesia in patients undergoing cataract surgery.

Keywords: Surgical anaesthesia; Topical anaesthesia; Subtenon anaesthesia; Cataract surgery

\section{Introduction}

Cataract remains one of the most common causes of visual impairment in the elderly [1]. In 2015, it was estimated that more than 20 million cataract surgeries were performed worldwide. These numbers roughly translate to about 400 eyes per year to operate on for a cataract surgeon [2]. With such numbers, time management efficiency is of utmost importance as the number of surgeries performed increases by the year. One of the more important advancements in reducing the time a patient spends on the operating table is the usage of topical anaesthesia. As much as it is important to reduce the time a patient spends in the operating theatre, patient experiences intraoperatively and postoperatively has to be similar if not better compared to other modes of administering anaesthesia such as through the subtenon or peribulbar routes.

Numerous studies have been done worldwide showing favourable results for topical anaesthesia in cataract surgery. In Malaysia, a comparative study was performed for the level of pain, visual perception and anxiety in 62 patients given either subtenon or intracameral anaesthesia [3]. They found no significant differences in the level of pain experiences between the 2 groups of patients. A number of other researches such as Zafirakis et al. in 2001 however have shown that the level of comfort of patients undergoing cataract surgery is lower compared to subtenon route of administration especially in the intraoperative and postoperative period [4].

We hope to prove that topical anaesthesia if administered properly is a comparable method of administering anaesthesia in patients undergoing cataract surgery.

\section{Methodology}

A total of 100 subjects planned for cataract surgery in the Ophthalmology Department of Hospital Sultan Haji Ahamd Shah (HoSHAS), Temerloh, Pahang were recruited from February 2016 to April 2016. These subjects underwent small incision phacoemulsification with intraocular lens implantation using either topical or sub-tenon's anaesthesia performed by a single surgeon. Approval for the study was obtained from the Medical Research and Ethics Committee, and Ministry Health Malaysia. Inclusion criteria were small incision cataract surgery and operation on the first eye. Exclusion criteria were subjects who had ocular morbidity apart from cataract, previous surgery in the eye to be operated, combined procedures and 
complicated cataract surgery. All subjects who participated in the study gave written informed consent.

Customized anaesthetic sets were prepared by the ophthalmology clinic nurses. Each anaesthetic set had a syringe containing either $3 \mathrm{ml}$ of lidocaine $2 \%(2 \mathrm{ml})$ and bupivacaine $0.75 \%(1 \mathrm{ml})$ or a placebo, and a dropper containing either proparacaine hydrochloride $0.5 \%$ or a placebo. These anaesthetic sets were numbered 1 to 100 . Each subject was allocated a number from 1 to 100 and received anaesthetic sets matching the number. All subjects received topical eye drops and a subtenon injection. They were randomized such that subjects receiving topical anaesthesia had a placebo subtenon injected, while subjects receiving subtenon injection had a topical placebo. The cataract surgeries were performed by a single surgeon using the phacoemulsification technique of divide and conquer. Neither the surgeon nor subjects involved were aware of the type of anaesthetic technique employed.

Depending on the group to which the subjects were randomized, they received either topical proparacaine hydrochloride $0.5 \%$ or a placebo of topical balanced salt solution. The method of administration was by a cotton bud soaked with either solution, placed locally in the lower conjunctival fornix over duration of 4 minutes before surgery before adding 5 drops of topical proparacaine hydrochloride $5 \%$ into the eye just before incision. For the sub-tenon's technique, proparacaine hydrochloride $0.5 \%$ drops were instilled. A small incision was made simultaneously in the conjunctiva and Tenon's capsule, about $5 \mathrm{~mm}$ from the limbus in the inferonasal quadrant, using curved Westcott scissors. A blunt 19-gauge curved cannula was then used to deliver approximately $3 \mathrm{ml}$ of $2 \%$ lignocaine, or $3 \mathrm{ml}$ of balanced salt solution in to the posterior sub-tenon's space.

Each subject was shown a visual analogue pain scale with numerical and descriptive ratings from 0 (no pain) to 10 (severe pain), to rate their pain. If any of the subjects were unable to read, a trained medical officer would explain the method of pain evaluation to them. Following administration of local anaesthesia, baseline pain scores were recorded in both groups. These pain scores were repeated during surgery, immediately on completion of surgery and 30 minutes postoperatively.

\section{Statistical Analysis}

Data obtained was entered into Microsoft excel and was analysed using the IBM SPSS Statistics Version 22.0. Normality of data was assessed with the Kolmogorov Smirnoff test. T test and chi square test was used. Pain score was tested with regression analysis. P-values $<0.05$ were considered to be statistically significant.

\section{Results}

A total of 118 subjects participated in this study. Forty subjects were randomized into the topical anaesthesia group and 78 to the subtenon anaesthesia group. There was no significant difference seen in the mean age of patients between the two groups (Figure 1a). $67.35 \pm 7.16$ in topical group versus $65.20 \pm 8.39$ in Subtenon group, $t$-test, $(P=0.16)$. There was also no significant difference in gender of patients in the two groups (Chi Square, $P=0.3$ ) (Figure 1b).

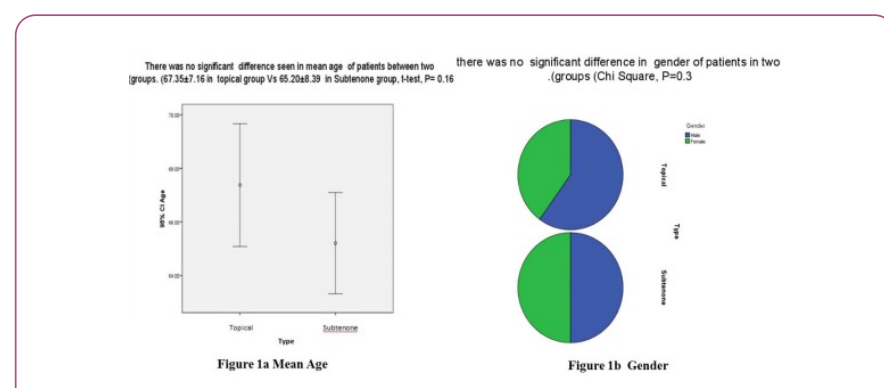

Figure 1 Patients' details in the topical and subtenon group.

There was no significant difference in mean pain score between two groups during anaesthesia $(P=0.48)$ (Figure 2a), during operation $(P=0.37)$ (Figure 2b), immediately after operation $(P=0.77)$ (Figure $2 c)$ and 30 minutes after operation $(P=0.17)$ (Figure 2d).

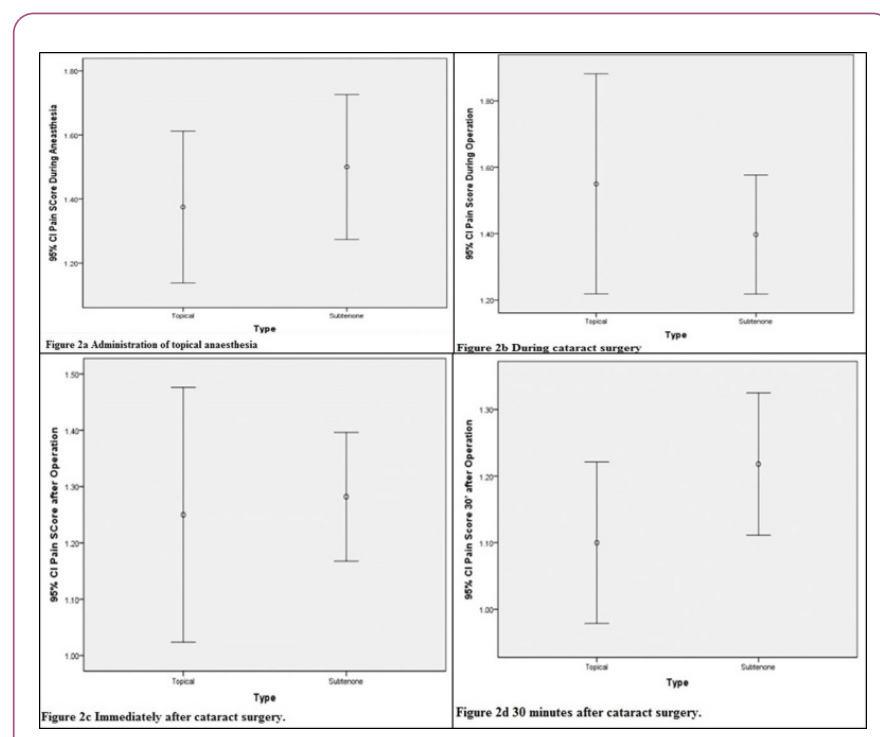

Figure 2 Pain score.

Multivariate analysis and regression model showed a significant relation of age with the pain score during anesthesia $(P=0.005)$ and 30 minutes after surgery $(P=0.038)$. There was a reverse significant correlation between age and pain score during anesthesia (Pearson Rho $=-0.29, P=0.001$ ), during operation (Pearson Rho=-0.20, $P=0.029$ ) and 30 minutes after operation (Pearson Rho $=-0.217, \mathrm{P}=0.018$ ).

\section{Discussion}

The search for the ideal anaesthesia has been enhanced by the emergence of topical anaesthetic agents. Most patients fear anaesthesia due to needles, intubation and the uncertain outcome of being under general anaesthesia. The eye is one of the most favourable sites in the body for application of 
successful topical anaesthesia due to a number of factors. The presence of unmyelinated nerve fibers in the cornea as well as a thin non-keratinized epithelium aids in the absorption of topical anaesthesia into the eye $[5,6]$. For these reasons, topical anaesthesia is widely used in cataract surgery. Waheeb et al. in 2010 reported of patient's and surgeon's satisfaction during cataract surgery with topical anaesthesia in a study conducted in 300 eyes [7]. Despite of numerous evidence on the efficacy of topical anesthesia as an alternative to subtenonor peribulbar injections, many surgeons are reluctant to use this method as their preferred choice for various reasons. Based on data from the Malaysian cataract surgery registry, it was found that the majority of eyes were operated under local anaesthesia [8]. They reported that more than $90 \%$ (93.6\% in 2002, 93.5\% in 2011) of the cases were done under local anesthesia. Subtenon injection was the preferred technique since 2002 but was surpassed by topical anaesthesia in 2010 (49.6\%) and 2011 (58.8\%). The change of preference towards topical anaesthesia mirrored the change toward phacoemulsification. A study was performed using a doubleblind randomized placebo control trial comparing topical and subtenon anaesthesia for cataract surgery reported that although cataract surgery was less painful under subtenon anaesthesia, topical anaesthesia was well tolerated by the subjects [9]. Our study found similar outcomes in terms of subject tolerance towards local anaesthesia. Since there were no significant differences in the pain scores of subjects during administration of anaesthesia, intra-operatively and after surgery in both groups whether they were given topical or subtenon's anaesthesia, topical anaesthesia provides comparable comfort to sub tenon's anaesthesia in cataract surgery.

\section{Conclusion}

The benefits of topical anaesthesia compared to subtenon's anaesthesia are also well documented. Topical anaesthesia saves the patients from risks of globe perforations, optic nerve injuries, possible life threatening respiratory arrest and the pain and fear perceived because of peribulbar or retrobulbar injections. It also benefits patients by not interfering with visual function, immediate visual recovery, absence of pain due to injection and unlimited ocular motility according to Nielsen in 1995 and Gupta et al. in 2009 [10,11]. Additional advantages are the absence of chemosis and subconjunctival hemorrhage, thereby improving the immediate post-operative cosmetic outcome of the patient according [12]. In a study done in the Netherlands, it was found that topical anaesthesia was more cost effective than subtenon anaesthesia in cataract surgery [13]. Topical anaesthesia also superceded peribulbar anaesthesia in cost effectiveness [14]. Taking into account the benefits, cost-effectiveness and ease of administration of topical anaesthesia as projected by various studies, it is proven to be an ideal anaesthetic method, comparable to subtenon anaesthesia for cataract surgery.

\section{References}

1. Buch H, Vinding T, Nielsen NV (2001) Prevalence and causes of visual impairment according to world health organization and United States criteria in an aged, urban scandinavian population: The copenhagen city eye study. Ophthalmology 108: 2347-2357.

2. Lindstrom R (2015) Thoughts on cataract surgery: 2015. Rev Opthalmol.

3. Mustapha M, Hamzah MH, Chow SK, Zulkifli NS, Latiff ZA, et al. (2014) Subtenon anaesthesia versus intracameral anaesthesia in patients undergoing cataract extraction: A comparative study of the level visual perception and anxiety. Int J Med Students 2: 49-52.

4. Zafirakis P, Voudouri A, Rowe S, Livir-Rallatos G, Livir-Allatos C, et al. (2001) Topical versus sub-tenon's anesthesia without sedation in cataract surgery. J Cataract Refract Surg 27: 873-879.

5. Obstbaum SA, Koch DD, Ford C, Pacheco W, Leo ES, et al. (1995) Cataract and Refractive Surgery.

6. Kumar M, Chawla R, Goyal M (2015) Topical anesthesia. J Anaesthesiol Clin Pharmacol 31: 450-456.

7. Waheeb S (2010) Topical anesthesia in phacoemulsification. Oman J Ophthalmo 3: 136-139.

8. Lee MY, Goh PP, Salowi MA, Adnan TH, Ismail M (2014) The malaysian cataract surgery registry: Cataract surgery practice pattern. Asia Pac J Ophthalmol (Phila) 3: 343-347.

9. Srinivasan S, Fern A, Selvaraj S, Hasan S (2004) Randomized double-blind clinical trial comparing topical and sub-tenon's anaesthesia in routine cataract surgery. Br J Anaesth 93: 683686.

10. Nielsen P J (1995) Immediate visual capability after cataract surgery: Topical versus retrobulbar anesthesia. J Cataract \& Refractive Surgery 21: 302-304.

11. Gupta SK, Kumar A, Kumar D, Agarwal S (2009) Manual small incision cataract surgery under topical anesthesia with intracameral lignocaine: Study on pain evaluation and surgical outcome. Indian J Ophthalmol 57: 3-7.

12. Roman SJ, Chong Sit DA, Boureau CM, Auclin FX, Ullern MM (1997) Sub-tenon's anaesthesia: An efficient and safe technique. Br J Ophthalmol 81: 673-676.

13. Brouwer JM (2010) Phd Thesis: Cheap Doesn't Always Mean Better: Anaesthesia in cataract extractions in the normal eye in the Netherlands; A deterministic cost utility analysis using a Markov Model. Umea ${ }^{\circ}$ International School of Public Health, Epidemiology, Global Health, Department of Public Health and Clinical Medicine, Umeå University, Sweden.

14. Guedes RP, Guedes VP, Chaoubah A, Pereira FM, Pereira PM, et al. (2011) Topical versus peribulbar anesthesia in nonpenetrating deep sclerectomy. A cost-effectiveness analysis. J Fr Ophtalmol 34: 629-633. 